\title{
Measurement of the Gluon Orbital Angular Momentum at the Electron-Ion Collider
}

\author{
Yong Zhao* \\ Center for Theoretical Physics, Massachusetts Institute of Technology, Cambridge, MA 02139, \\ USA \\ E-mail: yzhaoqcd@mit.edu
}

\section{Feng Yuan}

Nuclear Science Division, Lawrence Berkeley National Laboratory, Berkeley, CA 94720, USA

E-mail: fyuanelbl.gov

\section{Xiangdong Ji}

Tsung-Dao Lee Institute, and College of Physics and Astronomy, Shanghai Jiao Tong University, Shanghai, 200240, China

E-mail: xdjiesjtu.edu.cn

\begin{abstract}
The single longitudinal target-spin asymmetry in exclusive dijet production is sensitive to the gluon OAM density. The latter contributes to the spin asymmetry with a characteristic azimuthal angular modulation of $\sin \left(\phi_{\Delta}-\phi_{q}\right)$, where $\phi_{\Delta}$ and $\phi_{q}$ are the azimuthal angles of the proton momentum transfer and the relative transverse momentum between the quark-antiquark pair. This study will motivate a first measurement of the gluon OAM at the Electron-Ion Collider.
\end{abstract}

XXV International Workshop on Deep-Inelastic Scattering and Related Subjects 3-7 April 2017

University of Birmingham, $U K$

\footnotetext{
* Speaker.
} 


\section{Introduction}

Since the late 1960s great progress has been made in measuring the partonic content of proton spin, as results from SLAC, CERN, DESY, JLab and RHIC have nailed the quark spin contribution to about $30 \%[1,2,3]$. More recently, RHIC experiments have revealed that the gluon polarization contributes about $40 \%$ within the kinematic range of $0.05 \leq x \leq 0.2$ [4], which is an important part of the proton spin sum rule [5]. The orbital angular momenta (OAM) from the quarks and gluons play important roles in the partonic structure in nucleon, not only for the proton spin sum rule, but also for the novel phenomena in various high energy scattering processes. It has been shown in [6] that the total angular momentum contributions from the quarks and gluons can be studied through the associated generalized parton distributions (GPDs) [7, 8, 9] measured in the hard exclusive processes, such as the Deeply Virtual Compton Scattering (DVCS) [6, 8]. By subtracting the helicity contributions, we will be able to obtain the corresponding OAM contributions from the quarks and gluons.

Recent developments have also unveiled the close connection between the parton OAM and the associated quantum phase space distributions, the so-called Wigner distribution functions [10, $11,12,13,14]$,

$$
L_{q, g}(x)=\left.\varepsilon_{\perp}^{\alpha \beta} \frac{\partial}{i \partial \Delta_{\perp}^{\alpha}}\right|_{\Delta=0} \int d^{2} k_{\perp} k_{\perp}^{\beta} f_{q, g}\left(x, \xi, k_{\perp}, \Delta_{\perp}\right),
$$

where $f_{q, g}$ represent the quark/gluon Wigner distributions in a longitudinal polarized nucleon, and $\varepsilon_{\perp}^{\alpha \beta}$ represents 2-dimensional Levi-Civita symbol. We focus on the gluon Wigner distribution with light-cone gauge links and the corresponding OAM belongs to the Jaffe-Manohar spin sum rule $[15,16]$. The Wigner distributions are also referred to as the generalized transverse momentum dependent parton distributions (GTMD) [17].

To determine the GTMD in Eq. (1.1), we need to measure the correlation of $k_{\perp}$ and $\Delta_{\perp}$ from the observed final state particles. For $2 \rightarrow 2$ process, one can only have one independent momentum, so it is not likely for us to extrac the information we need, which leads us to consider $2 \rightarrow 3$ processes. We take the example of the single longitudinal target-spin asymmetries in hard exclusive dijet production in lepton-nucleon collisions [18],

$$
\ell+p \rightarrow \ell^{\prime}+q_{1}+q_{2}+p^{\prime}
$$

where the incoming and outgoing leptons have momenta $l$ and $l^{\prime}$, proton momenta with $p$ and $p^{\prime}$, and the final state two jets with momenta $q_{1}$ and $q_{2}$, as illustrated in Fig. 1. In high energy experiments at the EIC, the process of (2) is dominated by the gluon distribution from the target nucleon, and in particular, the differential cross section will depend on the gluon Wigner distribution [19]. Because of the relation of Eq. (1), one expects that the single longitudinal target-spin asymmetry of this process will be an ideal probe to the gluon OAM. To show this explicitly, we perform our calculations in a general collinear factorization framework, where the gluon OAM distribution enters at the twist-three level. The spin dependent differential cross section has a characteristic azimuthal angular dependence of $\sin \left(\phi_{\Delta}-\phi_{q}\right)$ where $\phi_{\Delta}$ and $\phi_{q}$ are the azimuthal angles of the proton momentum transfer and the relative transverse momentum between the quark pair as shown in Fig. 1. With a hermetic detector designed for the EIC, this observable can be well studied in the future, 


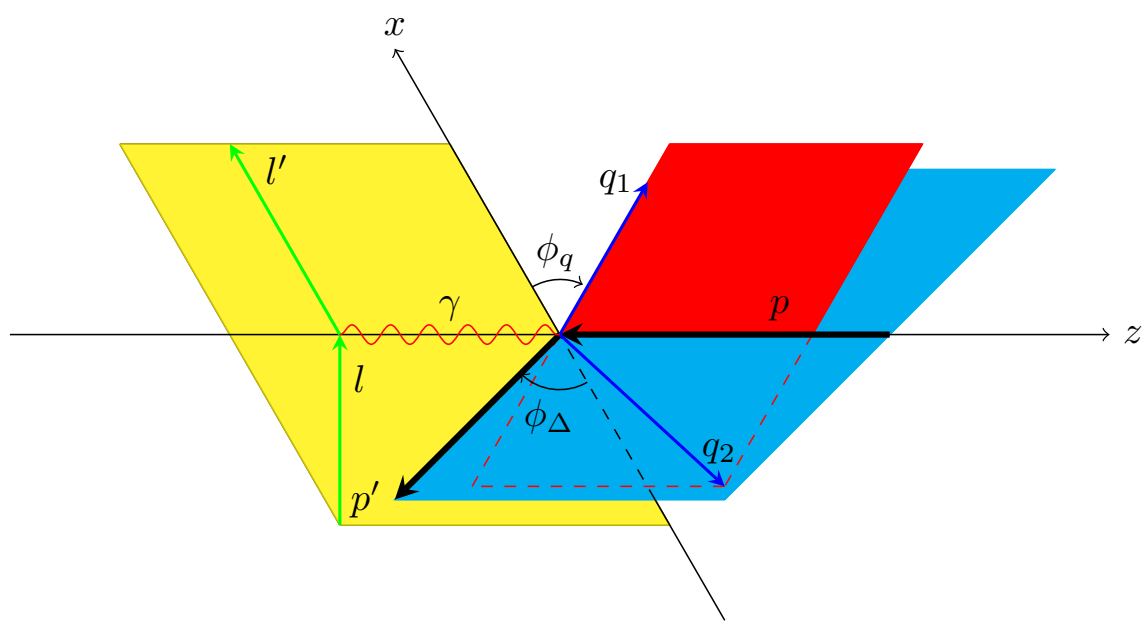

Figure 1: Hard exclusive dijet production in deep inelastic scattering to probe the gluon orbital angular momentum.

and will help us to finalize the proton spin sum rule, the ultimate goal for hadron physics in past decades.

The goal of this paper is to propose a direct measurement. Our approach and observables are also different from other proposals to measure the parton OAMs [20, 21]. In particular, we focus on the hard scattering processes which can be well studied at the planed EIC. The first results of this work has been reported in Ref. [22].

\section{Gluon OAM Contribution to the Single Spin Asymmetries}

The differential cross section of process (2) can be calculated through the lepton tensor and hadronic tensor,

$$
|\mathscr{M}|^{2}=L_{\mu v} H^{\mu v}
$$

where the lepton tensor takes a simple form of $L_{\mu \nu}=2\left(l_{\mu} l_{v}^{\prime}+l_{v} l_{\mu}^{\prime}-g_{\mu \nu} l \cdot l^{\prime}\right)$ due to the fact that the incoming lepton is unpolarized. The main task of our calculation is to evaluate the hadronic tensor, which comes from the Feynman diagrams illustrated in Fig. 2. We adopt the usual kinematics: the incoming photon with momentum $q=l-l^{\prime}, q^{2}=-Q^{2}, x_{B j}=Q^{2} /(2 q \cdot p), y=q \cdot p /(l \cdot p)$. The quark and antiquark momenta are further parameterized by their longitudinal momentum fractions $z$ and $\bar{z}=1-z$ as well as their transverse momenta $q_{\perp}-\Delta_{\perp} / 2$ and $-q_{\perp}-\Delta_{\perp} / 2$. In addition, for the exclusive processes, we have the following kinematics: $\Delta=p^{\prime}-p, P=\left(p+p^{\prime}\right) / 2, t=$ $\Delta^{2},(q+p)^{2}=W^{2},(q-\Delta)^{2}=\left(q_{1}+q_{2}\right)^{2}=M^{2}$, and the skewness parameter is defined as $\xi=$ $\left(p^{+}-p^{\prime+}\right) /\left(p^{+}+p^{+}\right)$with $p^{ \pm}=\left(p^{0} \pm p^{z}\right) / \sqrt{2}$, where $q$ and $p$ are chosen to be along the $z$ axis. As shown in Fig. 1, the lepton plane is set as the $x-z$ plane. The quark pair are in one plane with azimuthal angle $\phi_{q}$ respect to the lepton plane, whereas the recoiled proton is in another plane with momentum transfer $\vec{\Delta}_{\perp}$ and azimuthal angle $\phi_{\Delta}$. The spin-average cross section for this process has been calculated in Ref. [18]. 


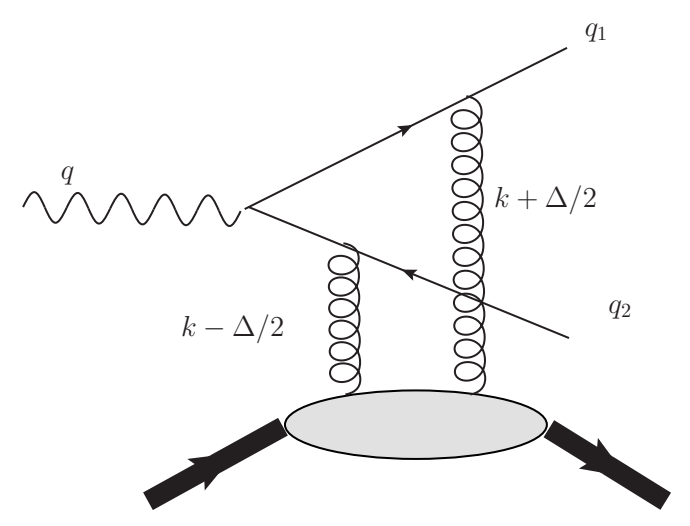

Figure 2: Generic Feynman diagram to evaluate the single longitudinal spin asymmetry in the hard exclusive dijet production in deep inelastic lepton nucleon scattering processes. All possible gluon attachment has been included in our calculations.

Following the usual collinear expansion at the next-to-leading power, we write the scattering amplitude, depicted in Fig. 2, as

$$
i \mathscr{A}_{f} \propto \int d x d^{2} k_{\perp} \mathscr{H}\left(x, \xi, q_{\perp}, k_{\perp}, \Delta_{\perp}\right) x f^{g}\left(x, \xi, k_{\perp}, \Delta_{\perp}\right),
$$

where $q_{\perp}$ is the jet transverse momentum defined above, and $k_{\perp}$ is the gluon transverse momentum entering the hard partonic part of Fig. 2. In this calculation, $q_{\perp}$ is the same order of $Q$, while the nucleon recoil momentum $\Delta_{\perp}$ is much smaller than $Q$. In the twist analysis, we expand the scattering amplitude in terms of $k_{\perp} / q_{\perp}$ (or $k_{\perp} / Q$ ),

$$
\mathscr{H}\left(x, \xi, q_{\perp}, k_{\perp}, \Delta_{\perp}\right)=\mathscr{H}^{(0)}\left(x, \xi, q_{\perp}, 0, \Delta_{\perp}\right)+k_{\perp}^{\alpha} \frac{\partial}{\partial k_{\perp}^{\alpha}} \mathscr{H}\left(x, \xi, q_{\perp}, 0, \Delta_{\perp}\right)+\cdots
$$

For the spin-average cross section, we take the zero-th order expansion of $k_{\perp}$. As a result, $k_{\perp}$ is integrated out for the gluon Wigner distribution,

$$
\int d^{2} k_{\perp} x f^{g}\left(x, \xi, k_{\perp}, \Delta_{\perp}\right)=F_{g}\left(x, \xi, \Delta_{\perp}\right),
$$

where $F_{g}$ is the spin-average gluon GPD. The scattering amplitude can be written as

$$
i \mathscr{A}_{f}^{(0)} \propto \int d x \mathscr{H}^{(0)}\left(x, \xi, q_{\perp}, 0,0\right) x F_{g}\left(x, \xi, \Delta_{\perp}\right) .
$$

Because $\Delta_{\perp} \ll q_{\perp}$, we have also taken $\Delta_{\perp}=0$ in the hard partonic part. This will enter into the spin-average cross section contribution, e.g., Eq. (2.7) below.

On the other hand, the single longitudinal target-spin asymmetry comes from the next-toleading power expansion of Eq. (2.3). Because of the nontrivial correlation between $k_{\perp}$ and $\Delta_{\perp}$ in the gluon Wigner distribution due to the gluon orbital motion, this contribution will lead to a novel correlation between $q_{\perp}$ and $\Delta_{\perp}$ as mentioned in Introduction,

$$
\int d^{2} k_{\perp}\left(\vec{q}_{\perp} \cdot \vec{k}_{\perp}\right) x f^{g}\left(x, \xi, k_{\perp}, \Delta_{\perp}\right)=-i S^{+}\left(\vec{q}_{\perp} \times \vec{\Delta}_{\perp}\right) x L_{g}\left(x, \xi, \Delta_{\perp}\right)+\cdots
$$


where we have only kept the spin-dependent matrix element in the above equation and $S^{+}$represents the longitudinal spin, and we have taken the leading contribution in terms of $\left(\vec{q}_{\perp} \cdot \vec{k}_{\perp}\right)$ in $\mathscr{H}$. We refer the above $L_{g}\left(x, \xi, \Delta_{\perp}\right)$ as the gluon OAM distribution, from which we shall be able to obtain the gluon OAM contribution to the proton spin from Eq. (1). According to this result, we only need to measure how the single target-spin asymmetry modulates with $\sin \left(\phi_{q}-\phi_{\Delta}\right)$ - which comes from $\left(\vec{q}_{\perp} \times \vec{\Delta}_{\perp}\right)$ - to extract the gluon OAM density.

For the spin-average cross section, we have the following expression [18],

$$
\frac{d \sigma}{d y d Q^{2} d \Omega}=\sigma_{0}\left[(1-y)\left|A_{L}\right|^{2}+\frac{1+(1-y)^{2}}{2}\left|A_{T}\right|^{2}\right],
$$

where $d \Omega$ represents the final hadronic states phase space: $d \Omega=d z d q_{\perp}^{2} d \Delta_{\perp}^{2} d \phi_{q \Delta} . \sigma_{0}$ is defined as

$$
\sigma_{0}=\frac{\alpha_{e m}^{2} \alpha_{s}^{2} e_{q}^{2}}{16 \pi^{2} Q^{2} y N_{c}} \frac{4 \xi^{2} z \bar{z}}{\left(1-\xi^{2}\right)\left(\vec{q}_{\perp}^{2}+\mu^{2}\right)^{3}},
$$

where $\mu^{2}=z \bar{z} Q^{2}$, and we have only kept the azimuthal angular symmetric terms in the above result and $\phi_{q \Delta}=\phi_{q}-\phi_{\Delta}$. The contributions from the transverse and longitudinal photons are: $\left|A_{L}\right|^{2}=$ $4 \beta\left|\mathscr{F}_{g}+4 \xi^{2} \bar{\beta} \mathscr{F}_{g}^{\prime}\right|^{2},\left|A_{T}\right|^{2}=\bar{\beta}(1 /(z \bar{z})-2)\left|\mathscr{F}_{g}+2 \xi^{2}(1-2 \beta) \mathscr{F}_{g}^{\prime}\right|^{2}$, where $\beta=\mu^{2} /\left(\mu^{2}+\vec{q}_{\perp}^{2}\right)$. We have defined the following generalized Compton form factors,

$$
\begin{aligned}
& \mathscr{F}_{g}(\xi, t)=\int d x \frac{1}{(x+\xi-i \varepsilon)(x-\xi+i \varepsilon)} F_{g}(x, \xi, t), \\
& \mathscr{F}_{g}^{\prime}(\xi, t)=\int d x \frac{1}{(x+\xi-i \varepsilon)^{2}(x-\xi+i \varepsilon)^{2}} F_{g}(x, \xi, t) .
\end{aligned}
$$

Following the above procedure, we derive the longitudinal target-spin dependent differential cross section [22],

$$
\frac{d \Delta \sigma}{d y d Q^{2} d \Omega}=\sigma_{0} \lambda_{p} \frac{2(\bar{z}-z)\left(\vec{q}_{\perp} \times \vec{\Delta}_{\perp}\right)}{\vec{q}_{\perp}^{2}+\mu^{2}}\left[(1-y) A_{f L}+\frac{1+(1-y)^{2}}{2} A_{f T}\right],
$$

where $\Delta \sigma=\left(\sigma\left(S^{+}\right)-\sigma\left(-S^{+}\right)\right) / 2$ and $\lambda_{p}$ represents the longitudinal polarization for the incoming nucleon. The spin-dependence comes from the interferences between the leading-twist and and twist-three amplitudes [22],

$$
\begin{aligned}
& A_{f L}=16 \beta \operatorname{Im}\left(\left[\mathscr{F}_{g}^{*}+4 \xi^{2} \bar{\beta} \mathscr{F}_{g}^{*}\right]\left[\mathscr{L}_{g}+8 \xi^{2} \bar{\beta} \mathscr{L}_{g}^{\prime}\right]\right) \\
& A_{f T}=2 \operatorname{Im}\left(\left[\mathscr{F}_{g}^{*}+2 \xi^{2}(1-2 \beta) \mathscr{F}_{g}^{\prime *}\right]\left[\mathscr{L}_{g}+2 \bar{\beta}\left(\frac{1}{z \bar{z}}-2\right)\left(\mathscr{L}_{g}+4 \xi^{2}(1-2 \beta) \mathscr{L}_{g}^{\prime}\right)\right]\right)
\end{aligned}
$$

where, again, we have defined the following Compton form factors to simplify the final results,

$$
\begin{aligned}
& \mathscr{L}_{g}(\xi, t)=\int d x \frac{x \xi}{(x+\xi-i \varepsilon)^{2}(x-\xi+i \varepsilon)^{2}} x L_{g}(x, \xi, t), \\
& \mathscr{L}_{g}^{\prime}(\xi, t)=\int d x \frac{x \xi}{(x+\xi-i \varepsilon)^{3}(x-\xi+i \varepsilon)^{3}} x L_{g}(x, \xi, t) .
\end{aligned}
$$

The above equations are the main results of our paper. Clearly, because of the pre-factor of Eq. (2.10), we find that the spin asymmetry is a power correction, which is consistent with our 
analysis. In addition, it is proportional to $\left(\vec{q}_{\perp} \times \vec{\Delta}_{\perp}\right)$, so that it has the characteristic azimuthal angular correlation $\sin \left(\phi_{q}-\phi_{\Delta}\right)$.

In order to observe the above spin asymmetry, we need to distinguish the two final state jets. This can be achieved by identifying the charge of the leading hadron in the jet, or by measuring the heavy quark pair through their decay products. For the latter process, we have to consider the mass effects, which can be straightforwardly taken into account. Similar calculations can be performed for the quark channel contributions, which may play important roles in the large- $x$ region. We will leave that for a future publication.

\section{Discussion}

As shown above, the gluon OAM contribution to the single longitudinal target-spin asymmetry has the novel azimuthal angular correlation of $\sin \left(\phi_{q}-\phi_{\Delta}\right)$. Experimentally, we have to identify the azimuthal angles of both $\vec{q}_{\perp}$ and $\vec{\Delta}_{\perp}$. In particular, it is challenging to precisely measure $\Delta_{\perp}$, because majority of the events will have small momentum transfer. Fortunately, the current design for the EIC detector will have excellent coverage to study the $\Delta_{\perp}$ distribution in the hard diffractive processes, including the proposed measurement of this paper, especially with the Roman Pot device along the beam line of the EIC. With the measurements of these two azimuthal angels, we can form the spin asymmetry,

$$
A_{\sin \left(\phi_{q}-\phi_{\Delta}\right)}=\int d \phi_{q} d \phi_{\Delta} \frac{d \sigma_{\uparrow}-d \sigma_{\downarrow}}{d \phi_{q} d \phi_{\Delta}} \sin \left(\phi_{q}-\phi_{\Delta}\right) / \int d \phi_{q} d \phi_{\Delta} \frac{d \sigma_{\uparrow}+d \sigma_{\downarrow}}{d \phi_{q} d \phi_{\Delta}} .
$$

From the results in the last section, we know that the above asymmetry will be sensitive to the gluon OAM distribution, and has the following kinematic dependence, schematically [22],

$$
A_{\sin \left(\phi_{q}-\phi_{\Delta}\right)} \propto \frac{(\bar{z}-z)\left|\vec{q}_{\perp}\right|\left|\vec{\Delta}_{\perp}\right|}{\vec{q}_{\perp}^{2}+\mu^{2}},
$$

where again, it is a twist-three effect. This asymmetry of the same process has also been discovered by a recent study on small- $x$ gluon OAM distribution [23], and is similar to the observable proposed in Ref. [24] to measure GTMD. The size of the asymmetry, of course, will depend on how large the gluon OAM is. Therefore, the experimental measurement of this asymmetry will provide direct access to the gluon OAM distribution. The unique angular correlation between the jet transverse momentum and the nucleon's recoiled momentum will help to identify the above asymmetry. We would like to emphasize that even if the asymmetry turns out small, it shall provide a strong constraint on the gluon OAM distribution. We are planing to have model predictions for the typical kinematics at the EIC, and hope this will lead to the first measurement of gluon OAM in the future.

Being a higher-twist effect, the asymmetry defined in Eq. (3.1) will have contributions from the twist-three multi-parton GPDs, in particular, those associated with three-gluon correlations $[15,16]$ shown in Fig. 3. For a complete evaluation of the single longitudinal target-spin asymmetries, we need to include these terms as well.

\section{Summary}

In summary, we have calculated the differential cross section for the hard exclusive electroproduction of quark-antiquark pair. The leading contribution to the single target-spin asymmetry 


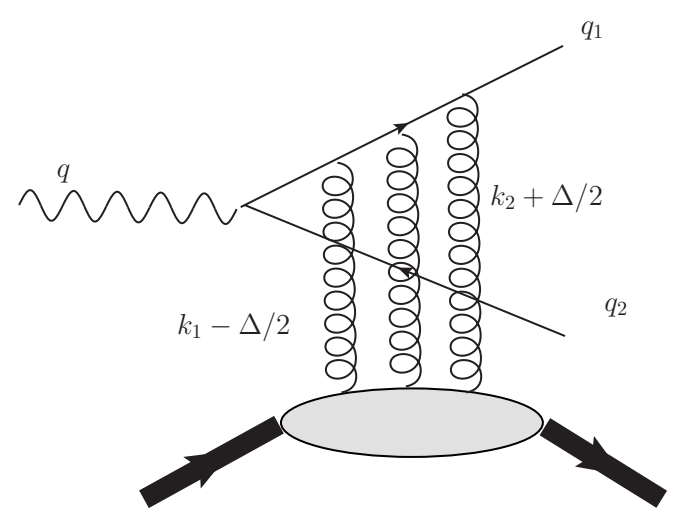

Figure 3: Feynman diagram that invloves three-gluon correlations. All other possible gluon attachments are also included.

is at order $1 / Q$, and crucially depends on the gluon OAM. In the kinematics covered by the EIC, this observable can be well studied, which will provide important information on the gluon OAM distribution in return.

In this paper, we focus on moderate $x$ range of the gluon OAM distribution. In the large$x$ region, we will also have quark-channel contributions, which can be used to probe the quark OAM. At small- $x$, on the other hand, we would expect the dipole framework is more appropriate to compute the process (2) [19]. However, the spin asymmetry is much more involved and nontrivial, which has been addressed Ref. [23].

\section{Acknowledgements}

We thank Elke-Caroline Aschenauer and Ernst Sichtermann for useful discussions on the experiment perspectives of the process studied in this paper. We also thank Yoshitaka Hatta and Yuya Nakagawa for comments and collaborations on the related subject [23]. This work was partially supported by the Laboratory Directed Research and Development Program of Lawrence Berkeley National Laboratory, the U.S. Department of Energy Office of Science, Office of Nuclear Physics under Award Number DE-FG02-93ER-40762 and DE-AC02-05CH11231, and a grant (No. 11DZ2260700) from the Office of Science and Technology in Shanghai Municipal Government, and also by grants from the National Science Foundation of China (No. 11175114, No. 11405104). YZ was also supported by the U.S. Department of Energy, Office of Science, Office of Nuclear Physics, from DE-SC0011090 and within the framework of the TMD Topical Collaboration.

\section{References}

[1] C. A. Aidala, S. D. Bass, D. Hasch and G. K. Mallot, Rev. Mod. Phys. 85, 655 (2013) doi:10.1103/RevModPhys.85.655 [arXiv:1209.2803 [hep-ph]]; and references therein.

[2] D. de Florian, R. Sassot, M. Stratmann and W. Vogelsang, Phys. Rev. D 80, 034030 (2009) doi:10.1103/PhysRevD.80.034030 [arXiv:0904.3821 [hep-ph]].

[3] E. R. Nocera et al. [NNPDF Collaboration], Nucl. Phys. B 887, 276 (2014) doi:10.1016/j.nuclphysb.2014.08.008 [arXiv:1406.5539 [hep-ph]]. 
[4] D. de Florian, R. Sassot, M. Stratmann and W. Vogelsang, Phys. Rev. Lett. 113, no. 1, 012001 (2014) doi:10.1103/PhysRevLett.113.012001 [arXiv:1404.4293 [hep-ph]].

[5] R. L. Jaffe and A. Manohar, Nucl. Phys. B 337, 509 (1990). doi:10.1016/0550-3213(90)90506-9

[6] X. D. Ji, Phys. Rev. Lett. 78, 610 (1997) doi:10.1103/PhysRevLett.78.610 [hep-ph/9603249].

[7] D. Mueller, D. Robaschik, B. Geyer, F.-M. Dittes and J. Hořejši, Fortsch. Phys. 42, 101 (1994) doi:10.1002/prop.2190420202 [hep-ph/9812448].

[8] X. D. Ji, Phys. Rev. D 55, 7114 (1997) doi:10.1103/PhysRevD.55.7114 [hep-ph/9609381].

[9] A. V. Radyushkin, Phys. Rev. D 56, 5524 (1997) doi:10.1103/PhysRevD.56.5524 [hep-ph/9704207].

[10] A. V. Belitsky, X. d. Ji and F. Yuan, Phys. Rev. D 69, 074014 (2004) doi:10.1103/PhysRevD.69.074014 [hep-ph/0307383].

[11] C. Lorce and B. Pasquini, Phys. Rev. D 84, 014015 (2011) doi:10.1103/PhysRevD.84.014015 [arXiv:1106.0139 [hep-ph]].

[12] Y. Hatta, Phys. Lett. B 708, 186 (2012) doi:10.1016/j.physletb.2012.01.024 [arXiv:1111.3547 [hep-ph]].

[13] C. Lorce, B. Pasquini, X. Xiong and F. Yuan, Phys. Rev. D 85, 114006 (2012) doi:10.1103/PhysRevD.85.114006 [arXiv:1111.4827 [hep-ph]].

[14] X. Ji, X. Xiong and F. Yuan, Phys. Rev. Lett. 109, 152005 (2012) doi:10.1103/PhysRevLett.109.152005 [arXiv:1202.2843 [hep-ph]].

[15] X. Ji, X. Xiong and F. Yuan, Phys. Rev. D 88, no. 1, 014041 (2013) doi:10.1103/PhysRevD.88.014041 [arXiv:1207.5221 [hep-ph]].

[16] Y. Hatta and S. Yoshida, JHEP 1210, 080 (2012) doi:10.1007/JHEP10(2012)080 [arXiv:1207.5332 [hep-ph]].

[17] S. Meissner, A. Metz and M. Schlegel, JHEP 0908, 056 (2009) doi:10.1088/1126-6708/2009/08/056 [arXiv:0906.5323 [hep-ph]].

[18] V. M. Braun and D. Y. Ivanov, Phys. Rev. D 72, 034016 (2005) doi:10.1103/PhysRevD.72.034016 [hep-ph/0505263].

[19] Y. Hatta, B. W. Xiao and F. Yuan, Phys. Rev. Lett. 116, no. 20, 202301 (2016) doi:10.1103/PhysRevLett.116.202301 [arXiv:1601.01585 [hep-ph]].

[20] A. Courtoy, G. R. Goldstein, J. O. G. Hernandez, S. Liuti and A. Rajan, Phys. Lett. B 731, 141 (2014) doi:10.1016/j.physletb.2014.02.017 [arXiv:1310.5157 [hep-ph]].

[21] A. Courtoy, G. R. Goldstein, J. O. Gonzalez Hernandez, S. Liuti and A. Rajan, arXiv:1412.0647 [hep-ph].

[22] X. Ji, F. Yuan and Y. Zhao, Phys. Rev. Lett. 118, no. 19, 192004 (2017) doi:10.1103/PhysRevLett.118.192004 [arXiv:1612.02438 [hep-ph]].

[23] Y. Hatta, Y. Nakagawa, F. Yuan, Y. Zhao and B. Xiao, Phys. Rev. D 95, no. 11, 114032 (2017) doi:10.1103/PhysRevD.95.114032 [arXiv:1612.02445 [hep-ph]].

[24] S. Bhattacharya, A. Metz and J. Zhou, Phys. Lett. B 771, 396 (2017) doi:10.1016/j.physletb.2017.05.081 [arXiv:1702.04387 [hep-ph]]. 